\title{
Gradient-Based Optimization using Parametric Sensitivity Macromodels
}

\author{
Krishnan Chemmangat*, Francesco Ferranti* ${ }^{*}$ Tom Dhaene*and Luc Knockaert* \\ Department of Information Technology, Ghent University - IBBT, Gaston Crommenlaan 8 Bus 201, B-9050, Gent, \\ Belgium.
}

\begin{abstract}
SUMMARY
A new method for gradient-based optimization of electromagnetic systems using parametric sensitivity macromodels is presented. Parametric macromodels accurately describe the parameterized frequency behavior of electromagnetic systems and their corresponding parameterized sensitivity responses with respect to design parameters, such as layout and substrate parameters. A set of frequency-dependent rational models is built at a set of design space points using the Vector Fitting method and converted into a statespace form. Then, this set of state-space matrices is parameterized with a proper choice of interpolation schemes, such that parametric sensitivity macromodels can be computed. These parametric macromodels, along with the corresponding parametric sensitivity macromodels, can be used in a gradient-based design optimization process. The importance of parameterized sensitivity information for an efficient and accurate design optimization is shown in the two numerical microwave examples. Copyright (c) 2011 John Wiley \& Sons, Ltd.

Received ...
\end{abstract}

KEY WORDS: Gradient-based Design Optimization; Parametric Sensitivity; Parametric Macromodeling; Interpolation.

\section{INTRODUCTION}

When designing high-speed microwave systems, one aims at obtaining the optimal values of the design variables for which the system responses satisfy the design specifications. This process is usually carried out through electromagnetic (EM) simulations. Optimal values of the design variables are often determined using optimization algorithms (optimizers) which drive the EM simulator to obtain the responses and their sensitivities in consecutive optimization iteration. Unfortunately, multiple consecutive EM simulations are often computationally expensive. An alternative approach is to generate accurate parametric macromodels up first, which capture the

\footnotetext{
${ }^{*}$ Correspondence to: Krishnan Chemmangat, Department of Information Technology, Ghent University - IBBT, Gaston Crommenlaan 8 Bus 201, B-9050, Gent, Belgium. E-mail: krishnan.cmc@intec.ugent.be

Contract/grant sponsor: This work was supported by the Research Foundation Flanders (FWO).

Copyright (c) 2011 John Wiley \& Sons, Ltd.
}

Prepared using jnmauth.cls [Version: 2010/03/27 v2.00] 
parameterized frequency behavior of the EM systems and their corresponding parameterized sensitivity responses with respect to design parameters, such as layout and substrate parameters. Efficient and accurate parametric sensitivity information is required by optimizers which employ state-of-the-art gradient-based techniques to calculate the optimal design parameters. Parametric sensitivity macromodels are able to describe sensitivity responses not only in the vicinity of a single operating point (local sensitivity), but over the entire design space of interest.

One of the most common approaches to calculate local sensitivities is the adjoint variable method. The main attractiveness of this approach is that sensitivity information can be obtained from at most two systems analyses regardless of the number of designable parameters [1, 2, 3]. However, these methods involve the calculation of the system matrix derivatives with respect to each design parameters, which is mostly done by finite difference approximations.

Recently, some interpolation-based parametric macromodeling techniques have been developed [4, 5, 6, 7, 8, 9], which interpolate a set of initial univariate macromodels, called root macromodels. In $[4,5,6]$, a parametric macromodel is built by interpolating a set of root macromodels at an inputoutput level, while in $[7,8,9]$ the interpolation process is applied to the internal state-space matrices of the root macromodels, therefore at a deeper level than in the transfer function-based interpolation approaches [4, 5, 6]. The methods [7, 8,9] allow to parameterize both poles and residues, hence their modeling capability is increased with respect to $[4,5,6]$, where only residues are parameterized.

A gradient-based minimax optimization process using parameterized sensitivity macromodels is presented in this paper. As in [7, 8, 9], an interpolation process on the internal state-space matrices of the root macromodels is performed. However, in $[7,8,9]$, the focus is on parametric macromodeling which ensures stability and passivity over the design space of interest. This is not strictly necessary for the calculation of parametric sensitivities, which allows the use of more powerful interpolation schemes which are at least continuously differentiable. The parametric sensitivity macromodels avoid the use of finite difference approximations in the optimization process. Also, in [7, 8, 9] computationally expensive linear matrix inequalities (LMI) are solved to guarantee preservation of passivity, which can be avoided in the present work. Parametric macromodels along with the corresponding parametric sensitivities are used in two pertinent numerical examples which confirm the applicability of the proposed technique to the optimization process of microwave filters. The importance of parameterized sensitivity information to speed up the design optimization process is shown in the pertinent examples.

\section{GENERATION OF ROOT MACROMODELS}

Starting from a set of data samples $\left\{(s, \vec{g})_{k}, \mathbf{H}(s, \vec{g})_{k}\right\}_{k=1}^{K_{t o t}}$, which depend on the complex frequency $s=j \omega$ and several design variables $\vec{g}=\left(g^{(n)}\right)_{n=1}^{N}$, such as layout features or substrate parameters, a set of frequency-dependent rational macromodels is built for some design space points by means of the Vector Fitting (VF) technique [10, 11, 12]. Each root macromodel has the following form:

$$
\mathbf{R}_{\vec{g}_{k}}(s)=\sum_{n=1}^{N_{P}} \frac{\mathbf{c}_{n}^{\vec{g}_{k}}}{s-a_{n}^{\vec{g}_{k}}}+\mathbf{d}^{\vec{g}_{k}}
$$




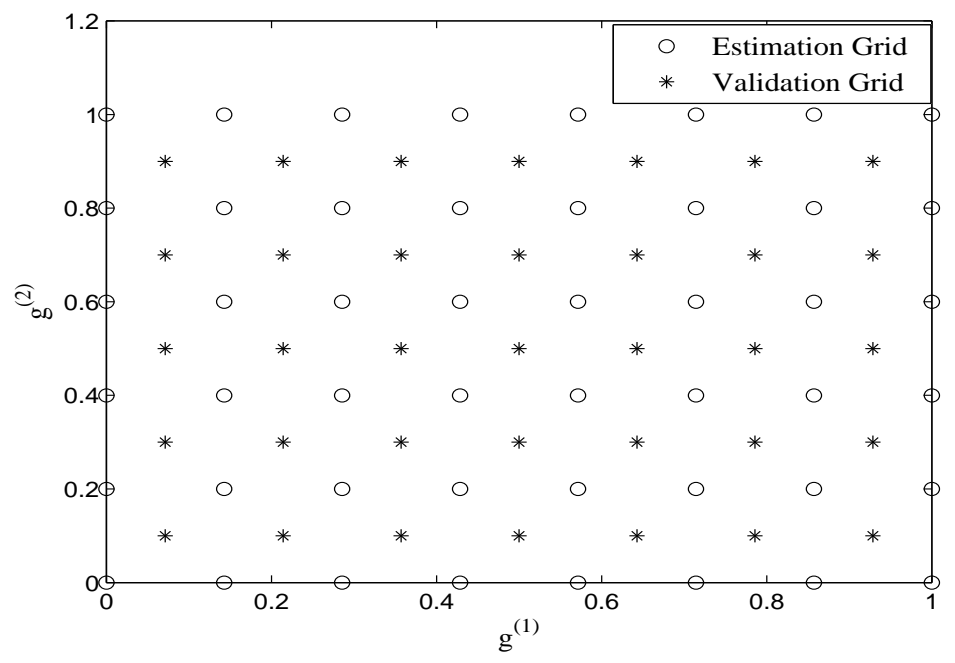

Figure 1. Estimation and Validation grids for a general two parameter design space.

The terms in the rational model (1), $a_{n}^{\vec{g}_{k}}, \mathbf{c}_{n}^{\vec{g}_{k}}$ and $\mathbf{d}^{\vec{g}_{k}}$ represent poles, residues and feed forward terms respectively at the design point $\vec{g}_{k}=\left(g_{k_{1}}^{(1)}, \ldots, g_{k_{N}}^{(N)}\right)$. The problem of finding the unknown coefficients in (1) is nonlinear, since the poles $a_{n}^{\vec{g} k}$ appear in the denominator. The idea of the VF technique is to recast this nonlinear identification problem into a linear least-square problem that is solved iteratively [10]. A pole-flipping scheme is used to enforce stability [10], while passivity assessment and enforcement can be accomplished using the standard techniques [13, 14]. This initial step of the proposed method results in a set of stable and passive frequency dependent rational models, called root macromodels.

Two design space grids are used in the modeling process: an estimation grid and a validation grid. The first grid is utilized to build the parametric macromodels. The second grid is utilized to assess the capability of parametric macromodels of describing the system under study in a set of points of the design space previously not used for their construction. To clarify the use of these two design space grids, we show in Fig. 1 a possible estimation and validation design space grid in the case of two design parameters $\vec{g}=\left(g^{(1)}, g^{(2)}\right)$. A root macromodel is built for each estimation grid point in the design space. This set of root macromodels is interpolated, as explained in the following section, to build a parameteric macromodel that is evaluated and compared with original data related to the validation design space points.

\section{PARAMETRIC MACROMODELING}

Each root macromodel $\mathbf{R}_{\vec{g}_{k}}(s)$, corresponding to a specific design space point $\vec{g}_{k}=\left(g_{k_{1}}^{(1)}, \ldots, g_{k_{N}}^{(N)}\right)$, is converted from the pole-residue form (1) into a state-space form:

$$
\mathbf{R}_{\vec{g}_{k}}(s)=\mathbf{C}_{\vec{g}_{k}}\left(s \mathbf{I}-\mathbf{A}_{\vec{g}_{k}}\right)^{-1} \mathbf{B}_{\vec{g}_{k}}+\mathbf{D}_{\vec{g}_{k}}
$$


Then, this set of state-space matrices $\mathbf{A}_{\vec{g}_{k}}, \mathbf{B}_{\vec{g}_{k}}, \mathbf{C}_{\vec{g}_{k}}, \mathbf{D}_{\vec{g}_{k}}$ is interpolated entry-by-entry and the multivariate models $\mathbf{A}(\vec{g}), \mathbf{B}(\vec{g}), \mathbf{C}(\vec{g}), \mathbf{D}(\vec{g})$ are built using multivariate interpolation schemes to generate a parametric macromodel $\mathbf{R}(s, \vec{g})$ for the entire design space [7, 8]:

$$
\mathbf{R}(s, \vec{g})=\mathbf{C}(\vec{g})(s \mathbf{I}-\mathbf{A}(\vec{g}))^{-1} \mathbf{B}(\vec{g})+\mathbf{D}(\vec{g}) .
$$

The computationally cheap piecewise linear interpolation can not be used to generate parametric sensitivity macromodels, since it is not continuously differentiable. A proper choice of interpolation schemes which are at least continuously differentiable is necessary. In this work, three interpolation methods are investigated, namely the cubic spline (CS) interpolation, the piecewise cubic Hermite interpolation (PCHIP) and the shape preserving C2 cubic spline interpolation (SPC2). They are briefly described in what follows.

\subsection{Cubic Spline Interpolation}

Given some data samples $\left(x_{i}, y_{i}\right)_{i=1}^{n}$, the CS interpolation method builds a cubic polynomial for each interval of the dataset $x_{i} \leq x \leq x_{i+1}, i=1, \ldots, n$.

$$
s_{i}(x)=a_{i}\left(x-x_{i}\right)^{3}+b_{i}\left(x-x_{i}\right)^{2}+c_{i}\left(x-x_{i}\right)+d_{i}
$$

The coefficients of the cubic polynomials are obtained by imposing the first and second order derivative continuity at each data point along with a not-a-knot end condition, and then solving a tridiagonal linear system [15]. Once these coefficients are computed, the derivatives of the overall spline interpolation function can be analytically calculated in terms of its coefficients $a_{i}, b_{i}$ and $c_{i}$ for $x_{i} \leq x<x_{i+1}, i=1,2, \ldots, n-1$. If the data under interpolation is in a matrix form, each entry of the matrices is independently interpolated.

The univariate CS interpolation can be extended to higher dimensions by means of a tensor product implementation [15].

\subsection{Piecewise Cubic Hermite Interpolation}

The PCHIP method is a monotonic shape preserving interpolation scheme. As in the CS interpolation, each data interval is modeled by a cubic polynomial similar to (4):

$$
p_{i}(x)=f_{i} H_{1}(x)+f_{i+1} H_{2}(x)+d_{i} H_{3}(x)+d_{i+1} H_{4}(x),
$$

where $d_{j}=\frac{d p\left(x_{j}\right)}{d x}, j=i, i+1$, and the $H_{k}(x)$ are the usual cubic Hermite basis functions for the interval $x_{i} \leq x<x_{i+1}, i=1,2, \ldots, n-1: H_{1}(x)=\phi\left(\left(x_{i+1}-x\right) / h_{i}\right), H_{2}(x)=\phi\left(\left(x-x_{i}\right) / h_{i}\right)$, $H_{3}(x)=-h_{i} \psi\left(\left(x_{i+1}-x\right) / h_{i}\right), H_{4}(x)=h_{i} \psi\left(\left(x-x_{i}\right) / h_{i}\right)$, where $h_{i}=x_{i+1}-x_{i}, \phi(t)=3 t^{2}-$ $2 t^{3}, \psi(t)=t^{3}-t^{2}$. The first order derivative $d_{i}$ at each data point $x_{i}$ is calculated such that the local monotonicity is preserved [16]. An extension to higher dimension can be performed by a tensor product implementation [15]. The calculation of derivatives is done in the same way as in the CS interpolation case. This interpolation scheme works better for non-smooth datasets, wherein the CS scheme could result in overshoots or oscillatory behavior of the derivatives. However, the PCHIP method is only continuous in first derivatives, which affects the smoothness of the derivatives [16]. 


\subsection{Shape Preserving C2 Cubic Spline Interpolation}

The SPC2 interpolation is a monotonicity preserving interpolation scheme similar to PCHIP. However, in contrast to the PCHIP method which is only continuous in first derivative, the SPC2 method is a second order derivative continuous interpolation scheme. The idea here is to add two extra break points in each subinterval of the data, such that enough degrees of freedom are generated to construct a cubic spline interpolant, which is globally second order derivative continuous [17]. Since the monotonicity of the data is preserved, this scheme works better with respect to the CS method for non-smooth data sets. Similar to the CS and the PCHIP interpolation schemes, a multivariate SPC2 interpolation is performed using a tensor product implementation [15].

\section{PARAMETRIC SENSITIVITY MACROMODELS}

Once the parametric macromodel $\mathbf{R}(s, \vec{g})$ is built, the corresponding sensitivities can be computed by differentiating (3) with respect to the design parameters $\vec{g}$

$$
\begin{array}{r}
\frac{\partial}{\partial \vec{g}} \mathbf{R}(s, \vec{g})=\frac{\partial \mathbf{C}(\vec{g})}{\partial \vec{g}}(s \mathbf{I}-\mathbf{A}(\vec{g}))^{-1} \mathbf{B}(\vec{g})+\mathbf{C}(\vec{g})(s \mathbf{I}-\mathbf{A}(\vec{g}))^{-1} \frac{\partial \mathbf{A}(\vec{g})}{\partial \vec{g}}(s \mathbf{I}-\mathbf{A}(\vec{g}))^{-1} \mathbf{B}(\vec{g})+ \\
\mathbf{C}(\vec{g})(s \mathbf{I}-\mathbf{A}(\vec{g}))^{-1} \frac{\partial \mathbf{B}(\vec{g})}{\partial \vec{g}}+\frac{\partial \mathbf{D}(\vec{g})}{\partial \vec{g}}
\end{array}
$$

In (6), $\frac{\partial}{\partial \vec{g}} \mathbf{R}(s, \vec{g})$ is based on the parameterized state-space matrices $\mathbf{A}(\vec{g}), \mathbf{B}(\vec{g}), \mathbf{C}(\vec{g}), \mathbf{D}(\vec{g})$ and the corresponding derivatives, which are computed efficiently and analytically using the interpolation methods described in Section 3.

\section{GRADIENT-BASED MINIMAX OPTIMIZATION}

Parametric sensitivity macromodels can be used in the optimization process of electromagnetic systems. Considering microwave filters, a typical optimization process begins by defining passband and stopband specifications in terms of the frequency responses, which are reformulated in the form of a cost function $F_{i}(\vec{g})$, at optimization frequency samples $s_{i}, i=1,2, . . N_{s}$ to be minimized:

$$
F_{i}(\vec{g})=R_{\mathrm{L}}^{i}-R\left(s_{i}, \vec{g}\right) \text { or } R\left(s_{i}, \vec{g}\right)-R_{\mathrm{U}}^{i}, \quad i=1,2, \ldots, N_{S}
$$

In (7), $R_{\mathrm{L}}^{i}$ and $R_{\mathrm{U}}^{i}$ represents lower and upper frequency response thresholds, respectively, at frequency samples $s_{i}$ spread over the frequency range of interest. A negative cost indicates that the corresponding specification is satisfied, while a positive cost denotes that the specification is violated. The minimization (7) can be performed by several state-of-the-art optimization algorithms. In this paper, we use a minimax optimization algorithm [18] which uses the cost function (7) and its gradients with respect to design parameter $\vec{g}$ giving the optimum design parameters $\overrightarrow{g^{*}}$ as,

$$
\overrightarrow{g^{*}}=\underset{\vec{g}}{\operatorname{argmin}}\left\{\max _{i}\left[F_{i}(\vec{g})\right]\right\}
$$




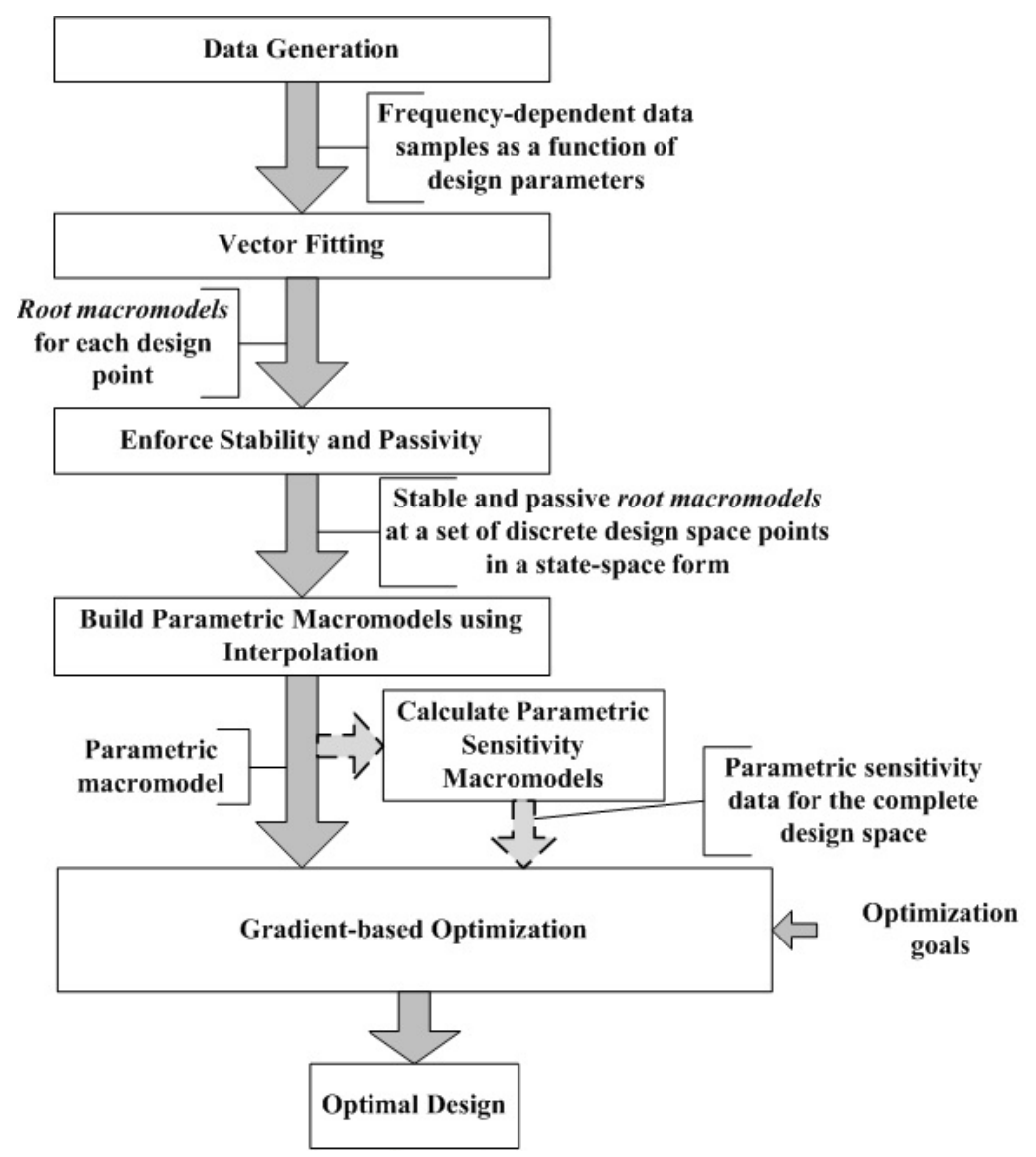

Figure 2. Complete optimization process flowchart.

The complete optimization process starting from the proposed parametric macromodeling technique is depicted in Fig. 2.

\section{NUMERICAL EXAMPLES}

\subsection{Double folded stub microwave filter}

A Double Folded Stub (DFS) microwave filter on a substrate with relative permitivity $\epsilon_{r}=9.9$ and a thickness of $0.127 \mathrm{~mm}$ is modeled in this example. The layout of this DFS filter is shown in Fig. 3. The spacing $S$ between the stubs and the length $L$ of the stub are chosen as design variables in addition to frequency. Their corresponding ranges are shown in Table I. The design specifications of this band-stop filter are given in terms of the scattering parameter, similarly to [19],

$$
\begin{array}{ll}
\left|S_{21}\right| \geq-3 \mathrm{~dB} \quad \text { for } \quad \text { freq } \leq 9 \mathrm{GHz} \text { or } \text { freq } \geq 17 \mathrm{GHz} \\
\left|S_{21}\right| \leq-30 \mathrm{~dB} \quad \text { for } \quad 12 \mathrm{GHz} \leq \text { freq } \leq 14 \mathrm{GHz}
\end{array}
$$




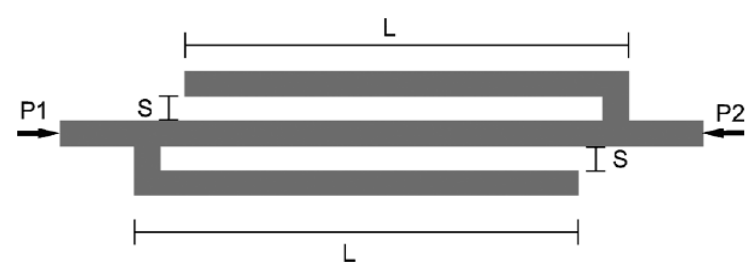

Figure 3. Layout of the DFS band-stop filter.

From the design specifications (9), a cost function (7) is formulated in terms of $S_{21}$ and $\vec{g}=(S, L)$.

Table I. Design parameters of the DFS band-stop filter

\begin{tabular}{|l|c|c|}
\hline Parameter & Min & Max \\
\hline Frequency $($ freq $)$ & $5 \mathrm{GHz}$ & $20 \mathrm{GHz}$ \\
Spacing $(S)$ & $0.1 \mathrm{~mm}$ & $0.25 \mathrm{~mm}$ \\
Length $(L)$ & $2.0 \mathrm{~mm}$ & $3.0 \mathrm{~mm}$ \\
\hline
\end{tabular}

The scattering matrix $\mathbf{S}(s, S, L)$ has been computed using the ADS Momentum ${ }^{\dagger}$ software. The $^{2}$ number of frequency samples were chosen to be equal to 31 . The estimation and validation grid points for the design variables are shown in Fig. 4. The average simulation time for each design space point $(S, L)$ has been found to be equal to, $T_{\text {Sim Avg }}=32.87 \mathrm{sec}$. A set of stable and passive root macromodels has been built for all design space points in the estimation grid of Fig. 4 by means of VF with a fixed number of poles $N_{P}=18$, selected using an error-based bottom-up approach. Each root macromodel has been converted into a state-space form and the set of state-space matrices has been interpolated using the CS, PCHIP and SPC2 methods. Let us define the absolute error

$$
\begin{aligned}
& \operatorname{Err}(\vec{g})=\max _{i, j, k}\left(\mid\left(R_{i, j}\left(s_{k}, \vec{g}\right)-H_{i, j}\left(s_{k}, \vec{g}\right) \mid\right)\right. \\
& i=1, \ldots, P_{\text {in }}, j=1, \ldots, P_{\text {out }}, k=1, \ldots, N_{s}
\end{aligned}
$$

where $P_{\text {in }}$ and $P_{\text {out }}$ are the number of inputs and outputs of the system, respectively, and $N_{s}$ is equal to the number of frequency samples. The worst case absolute error over the validation grid is chosen to assess the accuracy and the quality of parametric macromodels

$$
\begin{gathered}
\vec{g}_{\max }=\underset{\vec{g}}{\operatorname{argmax}} \operatorname{Err}(\vec{g}), \vec{g} \in \text { validation grid } \\
\operatorname{Err}_{\text {max }}=\operatorname{Err}\left(\vec{g}_{\text {max }}\right)
\end{gathered}
$$

\footnotetext{
${ }^{\dagger}$ Momentum EEsof EDA, Agilent Technologies, Santa Rosa, CA.
} 


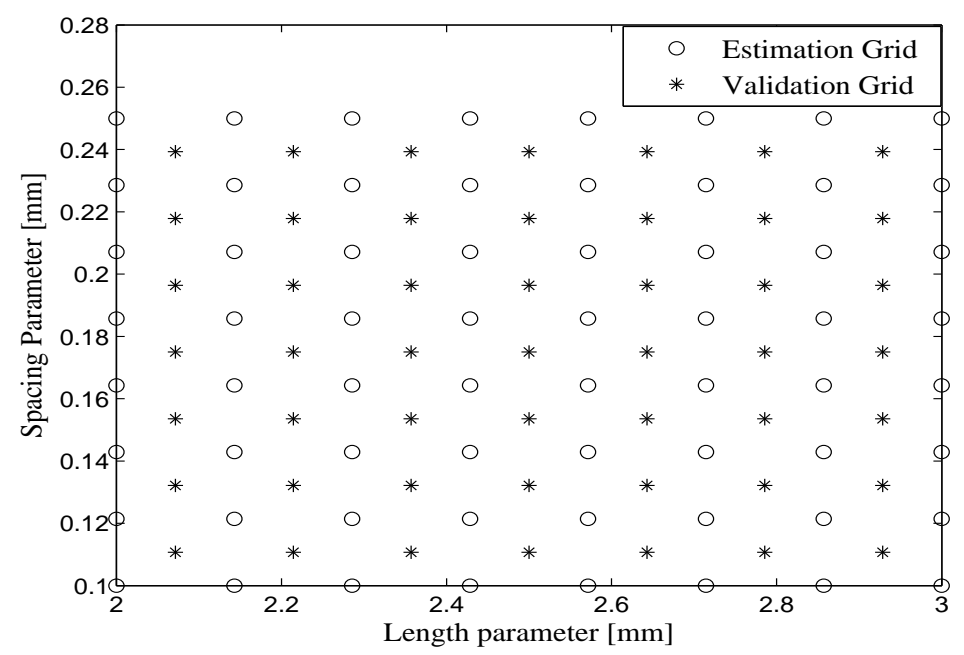

Figure 4. Estimation and Validation grids for the parametric macromodeling of the DFS Filter.

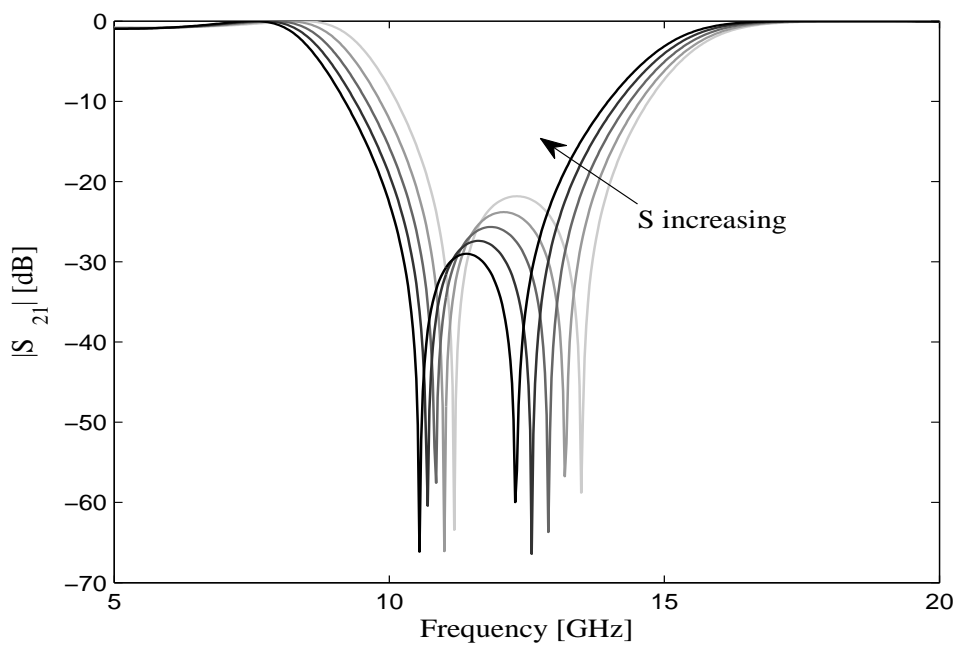

Figure 5. DFS filter: Magnitude of $S_{21}$ as a function of frequency for five values of $S$ and $L=2.5 \mathrm{~mm}$.

The maximum absolute error (12) for the parametric macromodel over the validation grid of Fig. 4 is $-58.45 \mathrm{~dB},-50.23 \mathrm{~dB}$ and $-50.23 \mathrm{~dB}$, respectively using the CS, PCHIP and SPC2 interpolation schemes. The CS interpolation scheme gives the minimum worst-case error (12) for this specific example and hence it has been used in the optimization process to generate the parametric macromodel and corresponding sensitivities. Fig. 5 shows the parametric behavior of the magnitude of $S_{21}$ as a function of frequency for five different values of $S$, and $L=2.5 \mathrm{~mm}$. In Fig. 5, the darkest curve of $S_{21}$ corresponds to the largest value of $S$. Similarly, Fig. 6 shows the magnitude of $S_{21}$ for five different values of $L$, with $S=0.175 \mathrm{~mm}$.

The cost function (7) and its gradients calculated using (6), have been supplied to the minimax optimization algorithm (8), resulting in the optimum design parameter values $S^{*}$ and $L^{*}$. To show 


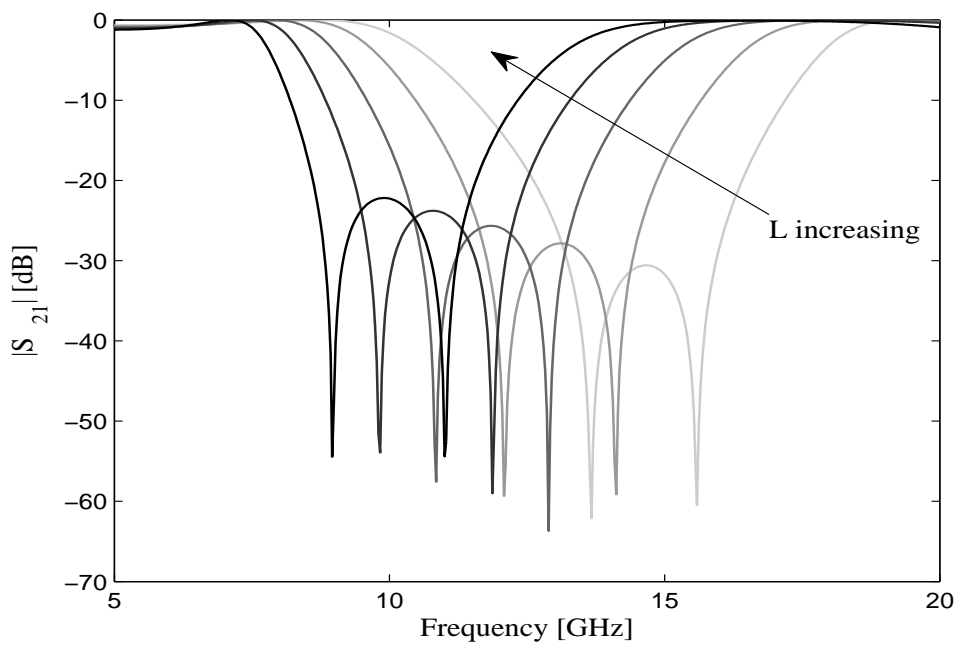

Figure 6. DFS filter: Magnitude of $S_{21}$ as a function of frequency for five values of $L$ and $S=0.175 \mathrm{~mm}$.

the advantage of supplying parametric sensitivity information to the optimizer to speed up the optimization process, two cases have been considered:

- Case I: No sensitivity information is supplied to the minimax algorithm and the algorithm estimates it with the help of finite difference approximation computed using the parametric macromodel.

- Case II: The sensitivity information is calculated from (6) and supplied to the minimax algorithm.

In addition to that, in order to show the advantage of using a parametric macromodel, the same optimization problem has been performed using the gradient-based minimax optimization routine in the ADS Momentum software. Table II compares these three optimization approaches in terms of optimization time for a particular optimization case. Table II shows the relevant speed-up in the optimization process obtained using the parametric macromodel. We note that the generation of the parametric macromodel requires some initial ADS Momentum simulations and therefore an initial computational effort of $3714.31 \mathrm{sec}$ (for the estimation and validation design space points in Fig. 4). However, once the parametric macromodel is generated and validated, it acts as an accurate and efficient surrogate of the original system and can be used for multiple design optimization scenarios, for instance, changing filter specifications. Fig. 7 shows the magnitude of $S_{21}$ for the optimization case of Table II. The actual data generated by the ADS Momentum software at the optimum design space point $\left(S^{*}, L^{*}\right)$ obtained using the parametric macromodel is shown by asterisks in Fig. 7. As seen, this is in good agreement with the parametric macromodel response. Fig. 8 shows the solution obtained using the gradient-based minimax optimization routine of ADS Momentum software. As clearly seen in Figs. 7-8, the optimal solutions satisfy all the requirements (9), which are shown by thin solid black lines. Fig. 9 shows the value of cost function with respect to the number of cost function evaluations in the Case I and II, which confirms the improved convergence of the optimization when parametric sensitivity information are provided. The convergence time taken by the Case I and II are $13.76 \mathrm{sec}$ and $1.73 \mathrm{sec}$, respectively. The trajectory of the optimum design space 
Table II. DFS filter: Optimization using parametric macromodel and ADS Momentum software.

\begin{tabular}{|c|c|c|c|c|c|}
\hline \multicolumn{2}{|c|}{ Method } & $\begin{array}{c}\left(S^{0}, L^{0}\right) \\
{[\mathrm{mm}]}\end{array}$ & $\begin{array}{c}\left(S^{*}, L^{*}\right) \\
{[\mathrm{mm}]}\end{array}$ & $\begin{array}{c}\text { Optimization } \\
\text { time [sec] }\end{array}$ & $\begin{array}{l}\text { Cost function at } \\
\left(S^{*}, L^{*}\right)[\mathrm{dB}]^{*}\end{array}$ \\
\hline Parametric & Case I & $(0.1500,2.6364)$ & $(0.2408,2.1802)$ & 19.88 & -1.67 \\
\hline macromodel & Case II & $(0.1500,2.6364)$ & $(0.2408,2.1802)$ & 1.23 & -1.67 \\
\hline \multicolumn{2}{|c|}{ ADS Momentum } & $(0.1500,2.6364)$ & $(0.2303,2.1580)$ & 5115.00 & 0.00 \\
\hline
\end{tabular}

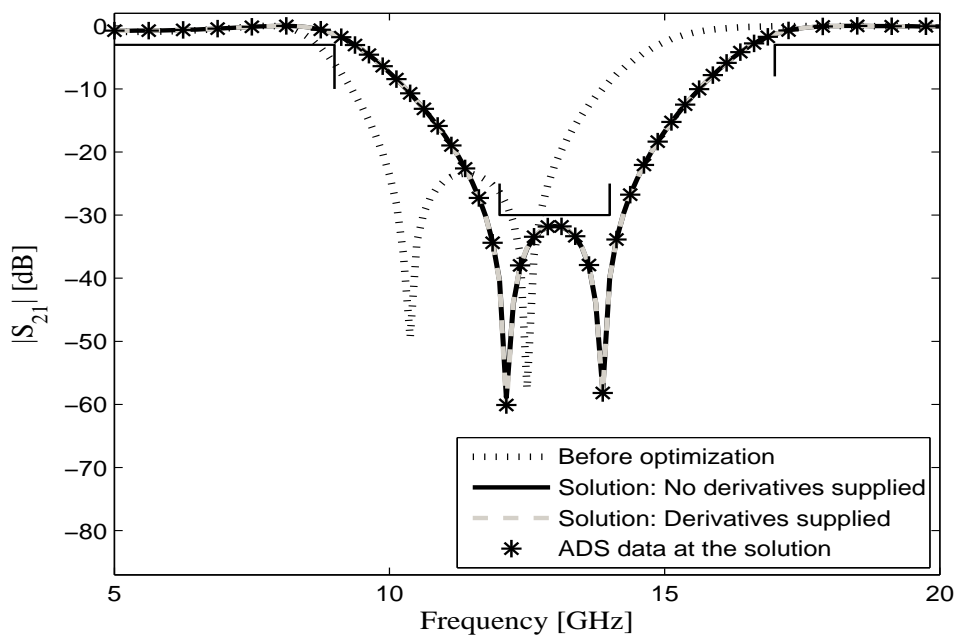

Figure 7. DFS filter: Magnitude of $S_{21}$ before and after optimization.

point $\left(S^{*}, L^{*}\right)$ during optimization for the Case I is shown in Fig. 10 with different points showing the output of some particular iterations along with the elapsed time. A similar curve is plotted in Fig. 11 for the Case II. Comparing Figs. 10-11, it is seen that the time for convergence of the Case II is considerably less compared with the Case I.

Table III shows the comparison of the Case I and II for some important optimization measures which are related to 200 optimization trials, starting from different initial design points spread over the design space. Table III confirms that, there is a considerable reduction in the number of cost function evaluations and the optimization time if derivatives are supplied (Case II).

\footnotetext{
*The Gradient-based minimax optimization routine of ADS Momentum software uses the minimax L1 error function which cannot take a value less than zero.
} 


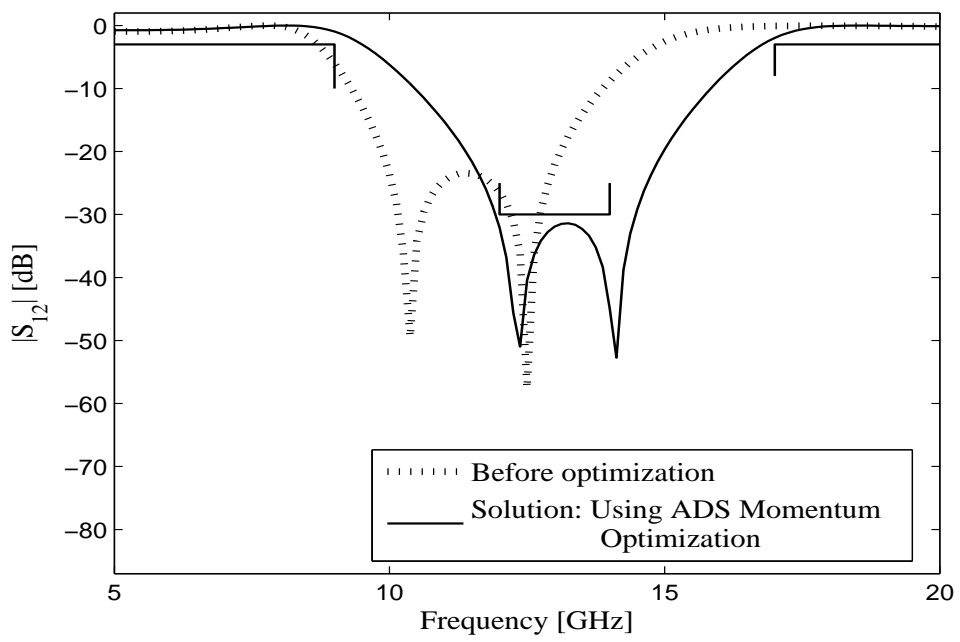

Figure 8. DFS filter: Magnitude of $S_{21}$ before and after optimization.

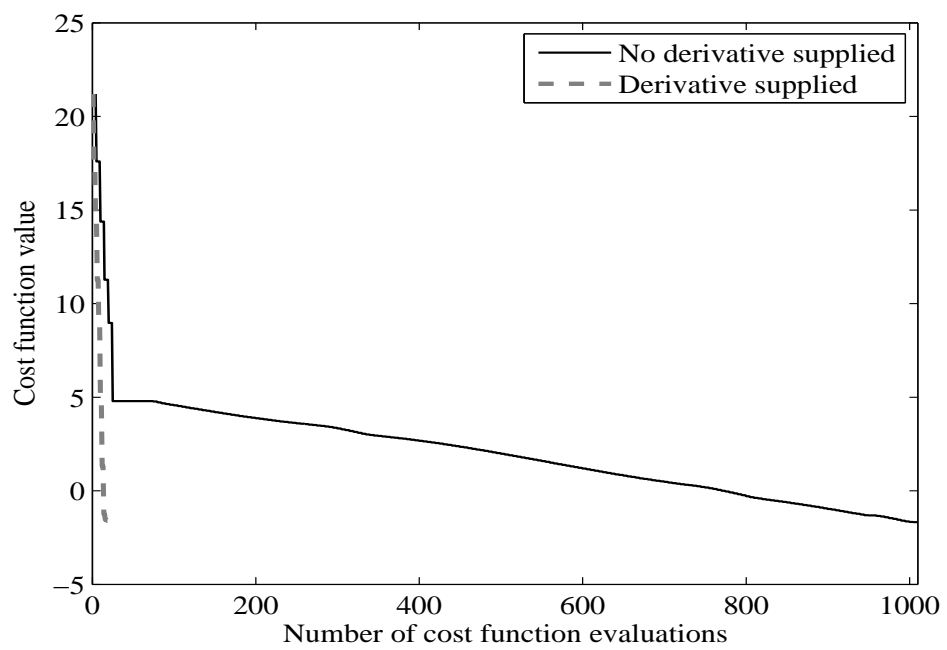

Figure 9. DFS filter: Cost function during optimization.

Table III. DFS filter: Comparison between the Cases I and II.

\begin{tabular}{|c|c|c|c|c|c|c|}
\hline Method & \multicolumn{3}{|c|}{ Number of cost function evaluations } & \multicolumn{3}{|c|}{ Optimization time [sec] } \\
\hline & Max & Mean & STD & Max & Mean & STD \\
\hline Case I & 20004 & 861.33 & 2360.10 & 135.30 & 6.05 & 16.31 \\
Case II & 1601 & 50.56 & 138.82 & 41.10 & 1.43 & 3.62 \\
\hline
\end{tabular}




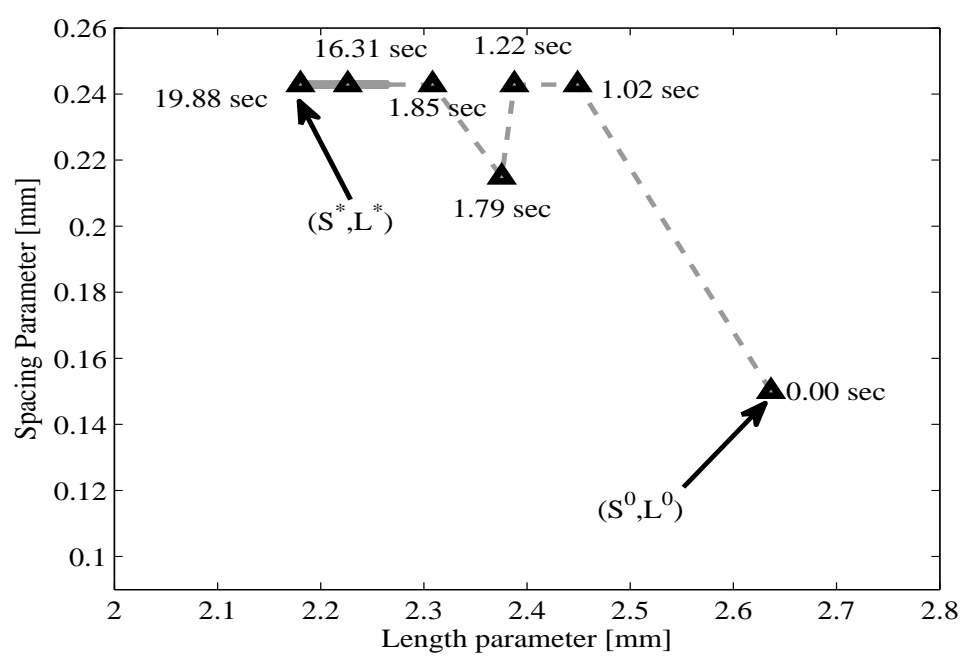

Figure 10. DFS filter: The trajectory of the optimal design space point $\left(S^{*}, L^{*}\right)$ during optimization for the Case I.

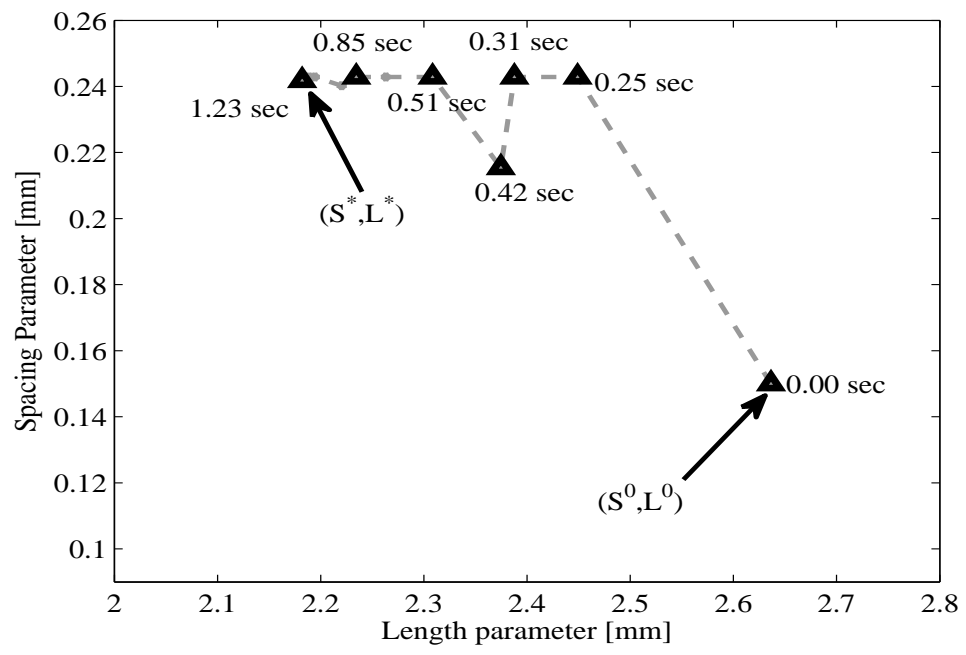

Figure 11. DFS filter: The trajectory of the optimal design space point $\left(S^{*}, L^{*}\right)$ during optimization for the Case II.

\subsection{Hairpin bandpass microwave filter}

In this example, a hairpin bandpass filter with the layout shown in Fig. 12 is modeled [19] . The relative permittivity of the substrate is $\epsilon_{r}=9.9$, while its thickness is equal to $0.635 \mathrm{~mm}$. The 


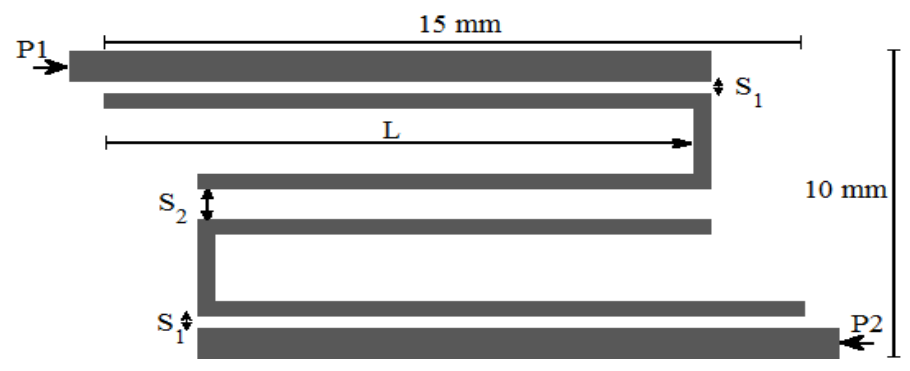

Figure 12. Layout of the Hairpin Bandpass Filter.

specifications for the bandpass filter are given in terms of the scattering parameters $S_{21}$ and $S_{11}$ :

$$
\begin{array}{lll}
\left|S_{21}\right|>-2.5 \mathrm{~dB} & \text { for } & 2.4 \mathrm{GHz}<\text { freq }<2.5 \mathrm{GHz} \\
\left|S_{11}\right|<-7 \mathrm{~dB} & \text { for } & 2.4 \mathrm{GHz}<\text { freq }<2.5 \mathrm{GHz} \\
\left|S_{21}\right|<-40 \mathrm{~dB} & \text { for } & \text { freq }<1.7 \mathrm{GHz} \\
\left|S_{21}\right|<-25 \mathrm{~dB} & \text { for } & 3.0 \mathrm{GHz}<\text { freq. }
\end{array}
$$

As shown in Fig. 12, three design parameters have been chosen for the design process namely, the spacing between the port lines and the filter lines $S_{1}$, the spacing between the two filter lines $S_{2}$ and the overlap length $L$ in addition to frequency. The ranges of the different design variables are shown in Table IV.

Table IV. Design parameters of the Hairpin Bandpass Filter

\begin{tabular}{|l|c|c|}
\hline Parameter & Min & Max \\
\hline Frequency $($ freq $)$ & $1.5 \mathrm{GHz}$ & $3.5 \mathrm{GHz}$ \\
Length $(L)$ & $12.0 \mathrm{~mm}$ & $12.5 \mathrm{~mm}$ \\
Spacing $\left(S_{1}\right)$ & $0.27 \mathrm{~mm}$ & $0.32 \mathrm{~mm}$ \\
Spacing $\left(S_{2}\right)$ & $0.67 \mathrm{~mm}$ & $0.72 \mathrm{~mm}$ \\
\hline
\end{tabular}

The scattering matrix $\mathbf{S}(s, S, L)$ has been computed using the ADS Momentum ${ }^{\ddagger}$ software. The number of frequency samples were chosen to be equal to 41 . As in the first example, two design space grids are used in the modeling process. The average simulation time for each design space point $\left(L, S_{1}, S_{2}\right)$ has been found to be equal to $T_{\text {Sim Avg }}=34.30 \mathrm{sec}$. A set of stable and passive root macromodels has been built for the estimation grid of $6 \times 4 \times 4\left(L \times S_{1} \times S_{2}\right)$ samples by means of VF with a fixed number of poles, $N_{P}=18$, selected using an error-based bottom-up approach. Each root macromodel has been converted into a state-space form and the set of state-space matrices has been interpolated by the CS, PCHIP and SPC2 methods. The maximum absolute error (12) of the models over the validation grid of $5 \times 3 \times 3\left(L \times S_{1} \times S_{2}\right)$ samples is equal to $-42.57 \mathrm{~dB},-38.27$ $\mathrm{dB}$ and $-38.27 \mathrm{~dB}$ for the CS, PCHIP and SPC2 methods, respectively. The CS technique has been

\footnotetext{
${ }_{\ddagger}^{\ddagger}$ Momentum EEsof EDA, Agilent Technologies, Santa Rosa, CA.
} 


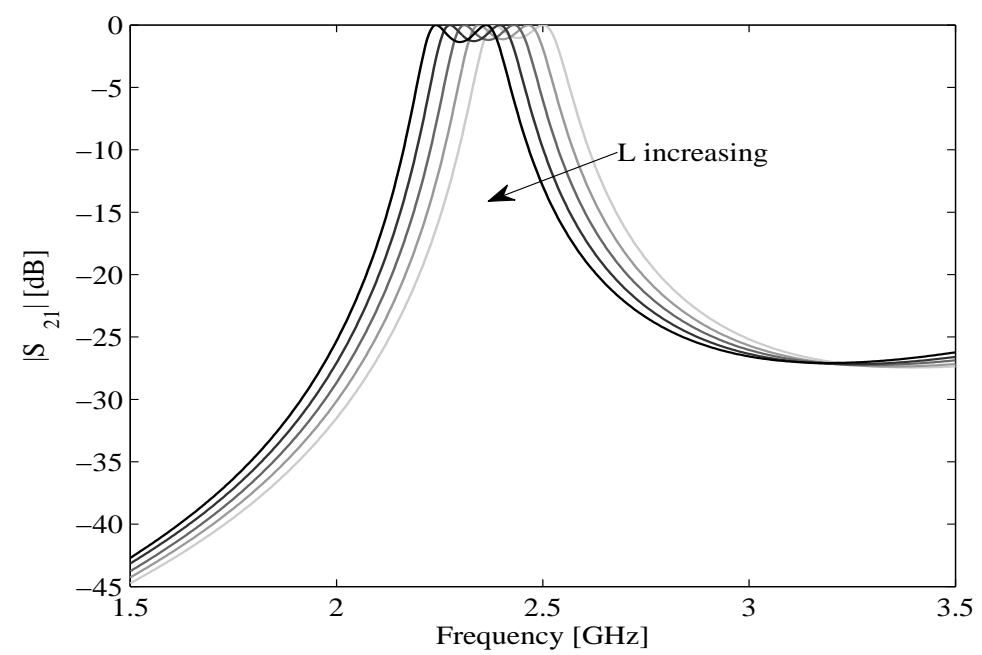

Figure 13. Hairpin filter: Magnitude of $S_{21}$ as a function of frequency for five values of $L$ with $\left(S_{1}, S_{2}\right)=$ $(0.295,0.695) \mathrm{mm}$.

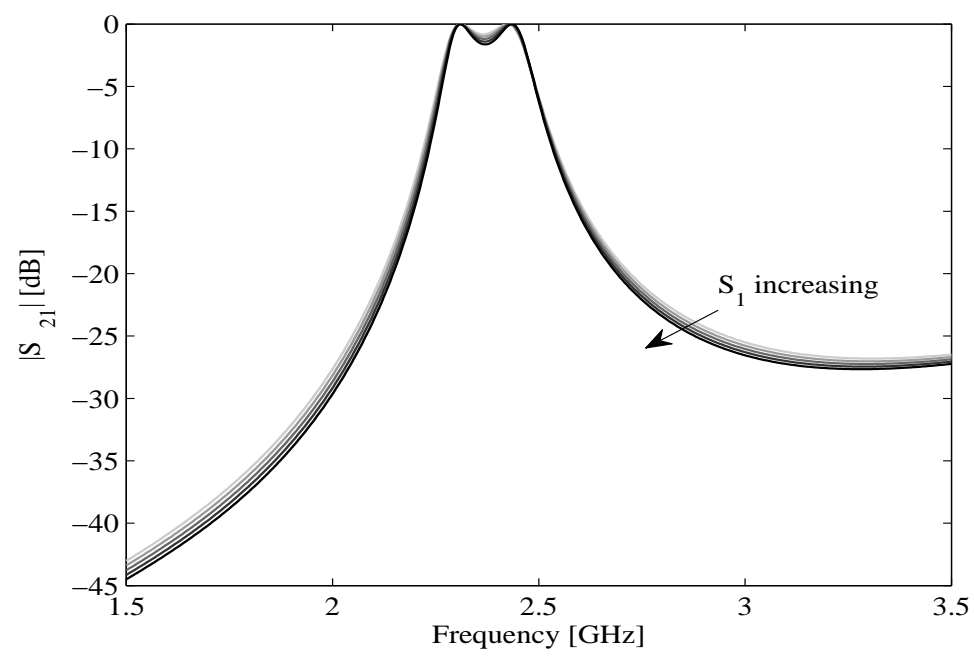

Figure 14. Hairpin filter: Magnitude of $S_{21}$ as a function of frequency for five values of $S_{1}$ with $\left(L, S_{2}\right)=$ $(12.25,0.695) \mathrm{mm}$.

used in the optimization process of this example, since it gives the best accuracy. Fig. 13 shows the parametric behavior of the magnitude of $S_{21}$ as a function of frequency for five different values of $L$ and $S_{1}=0.295 \mathrm{~mm}, S_{2}=0.695 \mathrm{~mm}$. Fig. 14 shows the magnitude of $S_{21}$ when the parameter $S_{1}$ changes.

The cost function (7) and its gradients calculated using (6), have been supplied to the minimax optimization algorithm (8), resulting in the optimum design parameter values $L^{*}, S_{1}^{*}$ and $S_{2}^{*}$. To show the improved convergence of the optimization when derivatives are supplied, two cases are considered as in the previous example. In addition to that, in order to show the advantage of using 


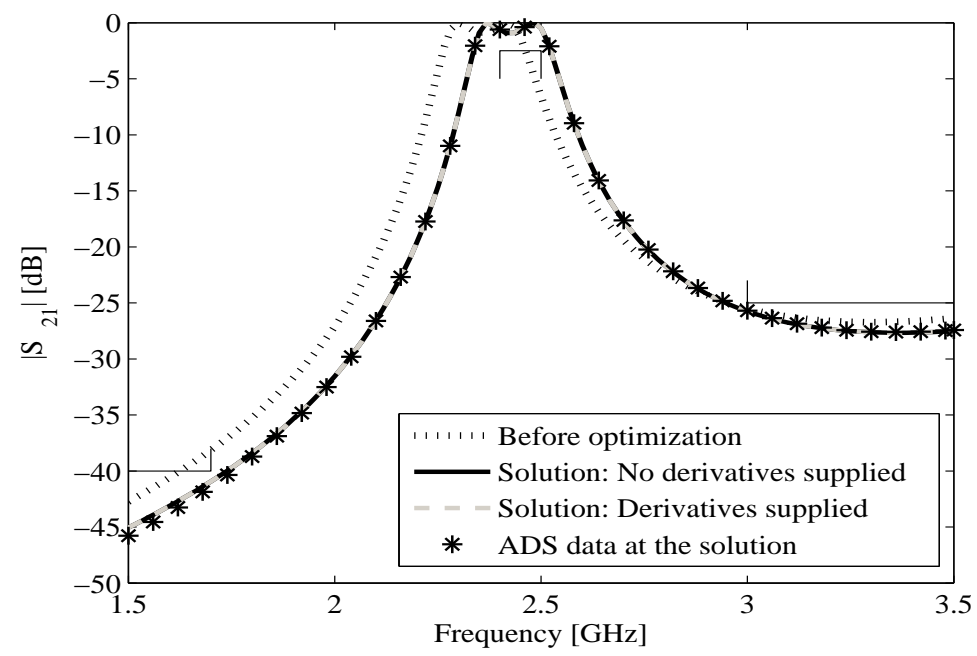

Figure 15. Hairpin filter: Magnitude of $S_{21}$ before and after optimization.

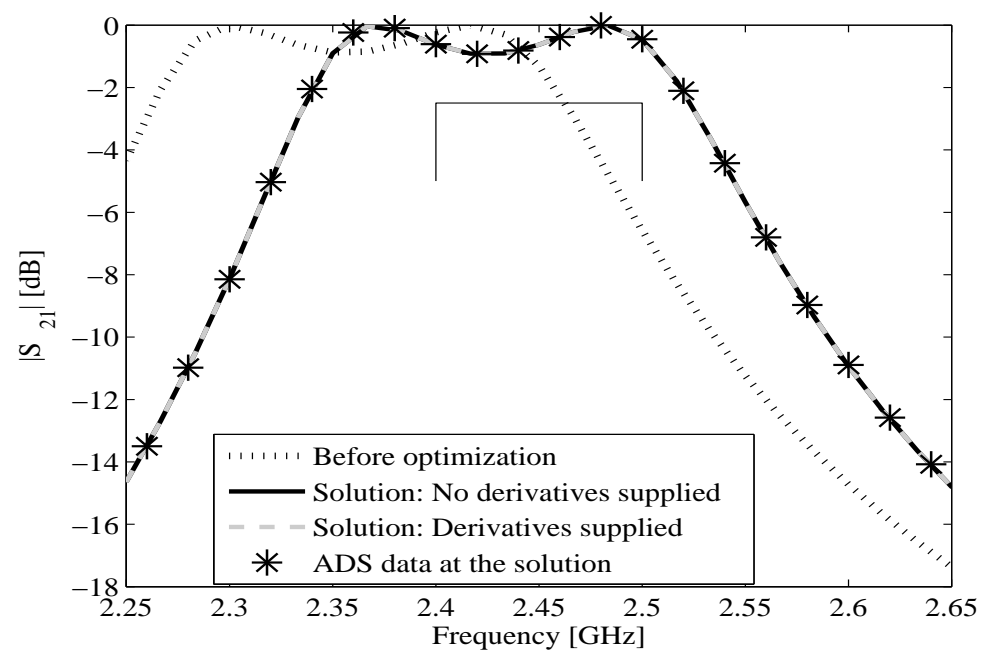

Figure 16. Hairpin filter: A zoomed in view of Fig. 15.

a parametric macromodel, the same optimization problem has been performed using the gradientbased minimax optimization routine in the ADS Momentum software. Table V compares these three optimization approaches in terms of optimization time for a particular optimization case. Table $\mathrm{V}$ shows the relevant speed-up in the optimization process obtained using the parametric macromodel. As mentioned in the previous example, the generation of the parametric macromodel requires an initial ADS Momentum simulation cost of $4836.30 \mathrm{sec}$. However, once the parametric macromodel is generated and validated, it acts as an accurate and efficient surrogate of the original system and can be used for multiple design optimization scenarios, for instance, changing filter specifications. Fig. 15 shows the magnitude of $S_{21}$ for the optimization case of Table. V. The actual data generated by the ADS Momentum software at the optimum design space point $\left(L^{*}, S_{1}^{*}, S_{2}^{*}\right)$ obtained using 


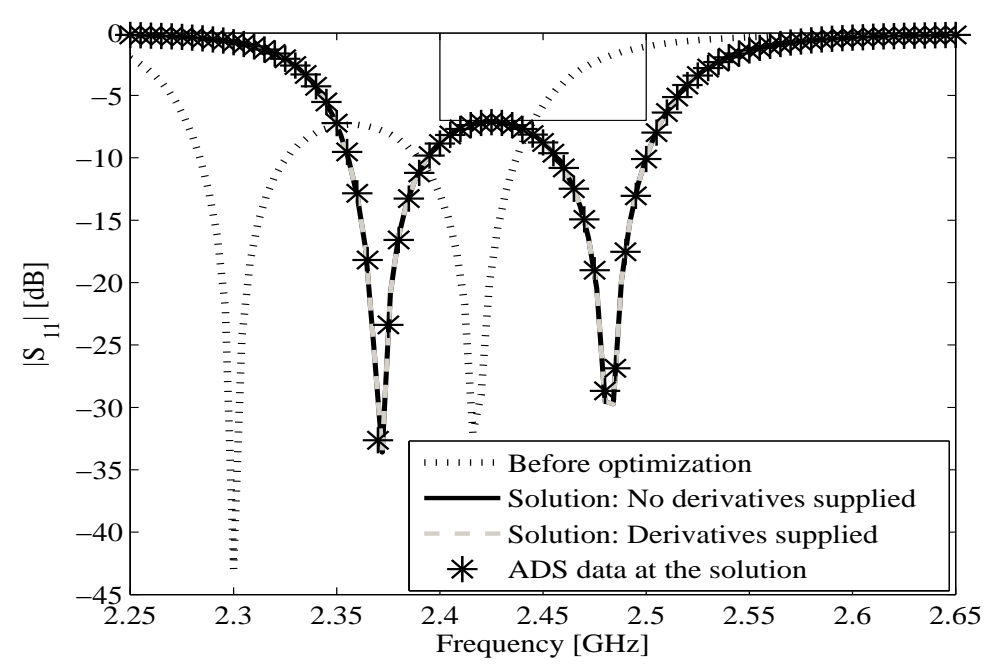

Figure 17. Hairpin filter: Magnitude of $S_{11}$ before and after optimization.

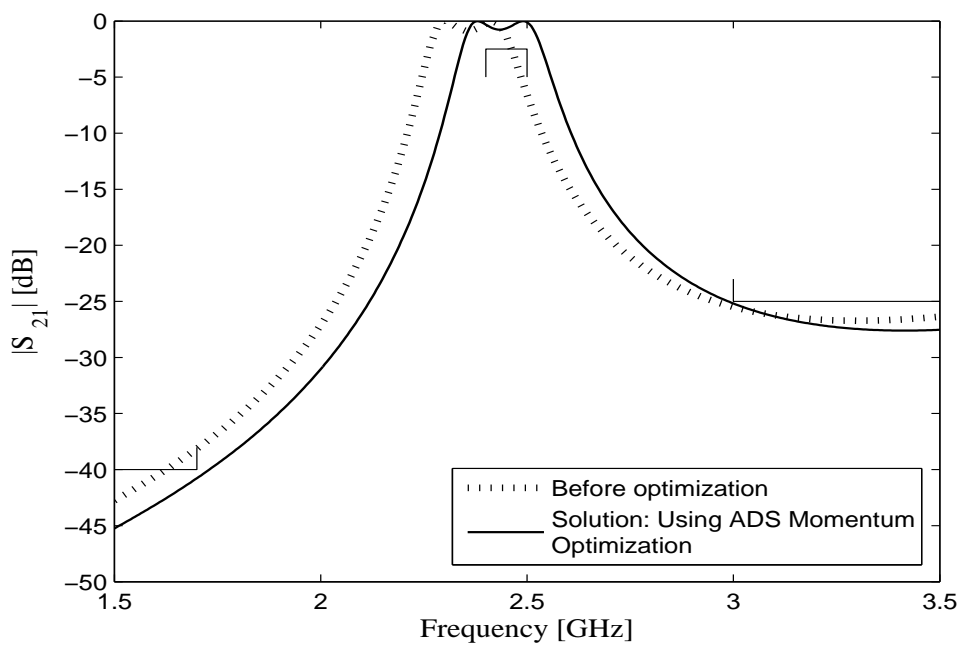

Figure 18. Hairpin filter: Magnitude of $S_{21}$ before and after optimization.

the parametric macromodel is shown by asterisks in Fig.15. As seen, this is in agreement with the parametric macromodel response. The requirements (13) are shown by the thin black solid lines. A magnified view of the passband is shown in Fig. 16 for clarity. Similar results are given for the magnitude of $S_{11}$ in Fig. 17. As clearly seen, all the requirements are met for the optimal design point. Figs. 18-19 shows similar results for the solution obtained using the gradient-based minimax optimization routine of ADS Momentum software. Here also, all the design specifications (13) are met. Some important measures of the optimization process related to 1000 trial runs are shown in Table VI, which clearly shows the better convergence properties of the Case II.

Another important thing to be noted here is that, with the increase in the number of design parameters the initial number of EM simulations increases considerably due to the curse of 


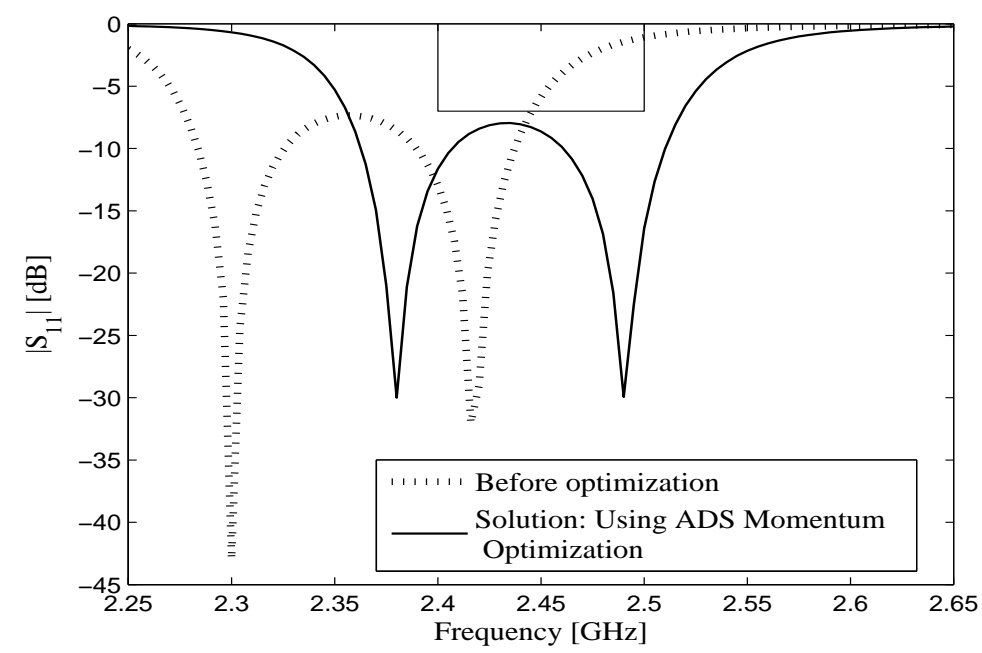

Figure 19. Hairpin filter: Magnitude of $S_{11}$ before and after optimization.

Table V. Hairpin filter: Optimization using parametric macromodel and ADS Momentum software.

\begin{tabular}{|c|c|c|c|c|c|}
\hline \multicolumn{2}{|c|}{ Method } & $\begin{array}{l}\left(L^{0}, S_{1}^{0}, S_{2}^{0}\right) \\
\quad[\mathrm{mm}]\end{array}$ & $\begin{array}{l}\left(L^{*}, S_{1}^{*}, S_{2}^{*}\right) \\
\quad[\mathrm{mm}]\end{array}$ & $\begin{array}{l}\text { Optimization } \\
\text { time[sec] }\end{array}$ & $\begin{array}{l}\text { Cost function at } \\
\left(L^{*}, S_{1}^{*}, S_{2}^{*}\right)[\mathrm{dB}]\end{array}$ \\
\hline Parametric & Case I & $\begin{array}{c}(12.2778,0.2700 \\
0.6867)\end{array}$ & $\begin{array}{c}(12.0568,0.2984 \\
0.7200)\end{array}$ & 13.46 & -0.76 \\
\hline macromodel & Case II & $\begin{array}{c}(12.2778,0.2700 \\
0.6867)\end{array}$ & $\begin{array}{c}(12.0568,0.2984 \\
0.7200)\end{array}$ & 0.79 & -0.76 \\
\hline \multicolumn{2}{|c|}{ ADS Momentum } & $\begin{array}{c}(12.2778,0.2700 \\
0.6867)\end{array}$ & $\begin{array}{c}(12.0036,0.2700 \\
0.6826)\end{array}$ & 1251.00 & 0.00 \\
\hline
\end{tabular}

Table VI. Hairpin filter: Comparison between the Cases I and II.

\begin{tabular}{|c|c|c|c|c|c|c|}
\hline Method & \multicolumn{2}{|c|}{ Number of cost function evaluations } & \multicolumn{3}{|c|}{ Optimization time [sec] } \\
\hline & Max & Mean & STD & Max & Mean & STD \\
\hline Case I & 53183 & 114.39 & 1689.71 & 565.14 & 1.29 & 17.95 \\
Case II & 99 & 13.73 & 5.79 & 4.69 & 0.76 & 0.28 \\
\hline
\end{tabular}

dimensionality. Adaptive sampling schemes, which take into account influence of the design parameters on the system behavior, could be used to properly sample the design space prior to the parametric macromodeling and help resolve this issue. For instance, in the second example, from Figs. 13 and 14 it is seen that the overlap length $L$ of the Hairpin Filter is more influential than the Spacing $S_{1}$, which allows one to sample along $S_{1}$ more sparsely using a wise adaptive sampling scheme, thereby reducing the number of initial EM simulations needed for the construction of parametric macromodels and the corresponding computational effort. 


\section{CONCLUSIONS}

Gradient-based design optimization of microwave systems using parametric sensitivity macromodels has been presented in this paper. Parameterized frequency-domain data samples are used to build a set of root macromodels in a state-space form. Then, this set of state-space matrices is parameterized using suitable interpolation schemes which are continuously differentiable. This allows to accurately and efficiently calculate parametric sensitivities over the entire design space of interest. Parametric macromodels and corresponding sensitivities are used for the gradientbased design optimization in two proposed numerical examples, which confirm the applicability of the proposed technique to the optimization process of microwave filters. Also, the importance of parameterized sensitivity information to speed up the design optimization process has been shown in the examples.

\section{REFERENCES}

1. Li D, Zhu J, Nikolova NK, Bakr MH, Bandler J. Electromagnetic optimisation using sensitivity analysis in the frequency domain. IET Microwaves, Antennas Propagation Aug 2007; 1(4):852 -859, doi:10.1049/iet-map: 20060303.

2. Dounavis A, Achar R, Nakhla M. Efficient sensitivity analysis of lossy multiconductor transmission lines with nonlinear terminations. IEEE Transactions on Microwave Theory and Techniques Dec 2001; 49(12):2292-2299, doi:10.1109/22.971612.

3. Nikolova NK, Bandler JW, Bakr MH. Adjoint techniques for sensitivity analysis in high-frequency structure CAD. IEEE Transactions on Microwave Theory and Techniques Jan 2004; 52(1):403-419, doi:10.1109/TMTT.2003. 820905.

4. Deschrijver D, Dhaene T. Stability and passivity enforcement of parametric macromodels in time and frequency domain. IEEE Transactions on Microwave Theory and Techniques Nov 2008; 56(11):2435 -2441, doi:10.1109/ TMTT.2008.2005868.

5. Ferranti F, Knockaert L, Dhaene T. Parameterized S-parameter based macromodeling with guaranteed passivity. Microwave and Wireless Components Letters, IEEE Oct 2009; 19(10):608 -610, doi:10.1109/LMWC.2009. 2029731.

6. Ferranti F, Knockaert L, Dhaene T. Guaranteed passive parameterized admittance-based macromodeling. IEEE Transactions on Advanced Packaging Aug 2010; 33(3):623 -629, doi:10.1109/TADVP.2009.2029242.

7. Ferranti F, Knockaert L, Dhaene T, Antonini G. Passivity-preserving parametric macromodeling for highly dynamic tabulated data based on Lur'e equations. Microwave Theory and Techniques, IEEE Transactions on Dec 2010; 58(12):3688 -3696, doi:10.1109/TMTT.2010.2083910.

8. Triverio P, Nakhla M, Grivet-Talocia S. Passive parametric macromodeling from sampled frequency data. 2010 IEEE 14th Workshop on Signal Propagation on Interconnects (SPI) May 2010; :117-120.

9. Triverio P, Nakhla M, Grivet-Talocia S. Passive parametric modeling of interconnects and packaging components from sampled impedance, admittance or scattering data. 3rd Electronic System-Integration Technology Conference (ESTC), Sept. 2010; 1 -6, doi:10.1109/ESTC.2010.5642961.

10. Gustavsen B, Semlyen A. Rational approximation of frequency domain responses by vector fitting. IEEE Transactions on Power Delivery July 1999; 14(3):1052-1061, doi:10.1109/61.772353.

11. Gustavsen B. Improving the pole relocating properties of vector fitting. IEEE Transactions on Power Delivery July 2006; 21(3):1587-1592, doi:10.1109/TPWRD.2005.860281.

12. Deschrijver D, Mrozowski M, Dhaene T, De Zutter D. Macromodeling of multiport systems using a fast implementation of the vector fitting method. IEEE Microwave and Wireless Components Letters June 2008; 18(6):1587-1592, doi:10.1109/LMWC.2008.922585.

13. Gustavsen B, Semlyen A. Fast passivity assessment for S-parameter rational models via a half-size test matrix. IEEE Transactions on Microwave Theory and Techniques Dec 2008; 56(12):2701-2708, doi:10.1109/TMTT.2008. 2007319. 
14. Gustavsen B. Fast passivity enforcement for S-parameter models by perturbation of residue matrix eigenvalues. IEEE Transactions on Advanced Packaging Feb 2010; 33(1):257-265, doi:10.1109/TADVP.2008.2010508.

15. de Boor C. A Practical Guide to Splines. Springer-Verlag New York, Inc.: New York, USA, 2001.

16. Fritsch FN, Carlson RE. Monotone piecewise cubic interpolation. SIAM Journal on Numerical Analysis Apr 1980; 17(2):238-246.

17. Pruess S. Shape preserving C2 cubic spline interpolation. IMA Journal on Numerical Analysis 1993; 13(4):493507.

18. Du DZ, Pardalos PM. Minimax and Applications. Kluwer Academic Publishers: Dordrecht, The Netherlands, 1995.

19. Ureel J, De Zutter D. Gradient-based minimax optimization of planar microstrip structures with the use of electromagnetic simulations. International Journal of Microwave and Millimeter-Wave Computer-Aided Engineering Jan 1997; 7(1):29-36.

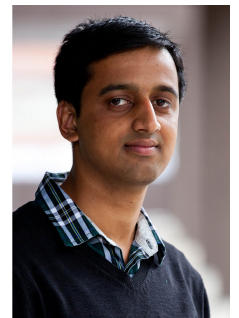

Krishnan Chemmangat was born in Kerala, India, on December 28, 1984. Krishnan received the Bachelor of Technology (B.Tech) degree in Electrical and Electronics engineering from the University of Calicut, Kerala, India in 2006 and the Master of Technology (M.Tech) degree in Control and Automation from the Indian Institute of Technology Delhi, New Delhi, India in 2008. Since April 2010 he is active as a $\mathrm{PhD}$ student in the research group Internet Based Communication Networks and Services (IBCN) at Ghent University. His research interests include parametric macromodeling, parameterized model order reduction, system identification.

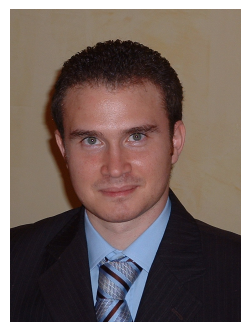

Francesco Ferranti received the B.S. degree (summa cum laude) in electronic engineering from the Universit degli Studi di Palermo, Palermo, Italy, in 2005, the M.S. degree (summa cum laude and honors) in electronic engineering from the Universit degli Studi dell'Aquila, L'Aquila, Italy, in 2007, and the Ph.D. degree in electrical engineering from the University of Ghent, Ghent, Belgium, in 2011. He is currently a Post-Doctoral Research Fellow with the Department of Information Technology (INTEC), Ghent University, Ghent, Belgium. His research interests include parametric macromodeling, parameterized model order reduction, electromagnetic compatibility numerical modeling, system identification.

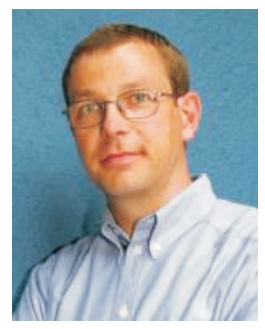

Tom Dhaene was born in Deinze, Belgium, on June 25, 1966. He received the Ph.D. degree in electrotechnical engineering from the University of Ghent, Ghent, Belgium, in 1993. From 1989 to 1993, he was Research Assistant at the University of Ghent, in the Department of Information Technology, where his research focused on different aspects of full-wave electro-magnetic circuit modeling, transient simulation, and time-domain characterization of high-frequency and high-speed interconnections. In 1993, he joined the EDA company Alphabit (now part of Agilent). He was one of the key developers of the planar EM simulator ADS Momentum. Since September 2000, he has been a Professor in the Department of Mathematics and Computer Science at the University of Antwerp, Antwerp, Belgium. Since October 2007, he is a Full Professor in the Department of Information Technology (INTEC) at Ghent University, Ghent, Belgium. As author or co-author, he has contributed to more than 150 peer-reviewed papers and abstracts in international conference proceedings, journals and books. He is the holder of 3 US patents. 


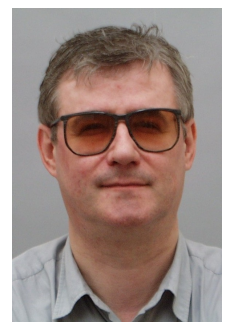

Luc Knockaert received the M. Sc. Degree in physical engineering, the M. Sc. Degree in telecommunications engineering and the Ph. D. Degree in electrical engineering from Ghent University, Belgium, in 1974, 1977 and 1987, respectively. From 1979 to 1984 and from 1988 to 1995 he was working in North-South cooperation and development projects at the Universities of the Democratic Republic of the Congo and Burundi. He is presently affiliated with the Interdisciplinary Institute for BroadBand Technologies (www.ibbt.be) and a professor at the Dept. of Information Technology, Ghent University (www.intec.ugent.be). His current interests are the application of linear algebra and adaptive methods in signal estimation, model order reduction and computational electromagnetics. As author or co-author he has contributed to more than 100 international journal and conference publications. He is a member of MAA, SIAM and a senior member of IEEE. 